of immunity from rubella vaccines. And the same uncertainty applies to mumps. This is another common infectious disease of childhood which may, like rubella, occur also in the adult. Among the complications of the disease the most frequent are lesions of the central nervous system, but fortunately most of these cases are of aseptic meningitis and the prognosis is usually good, in contrast to the severe encephalitis associated with measles. Orchitis, another well-known complication, is rarely bilateral, and the belief that it may lead to sterility is based more on fear and pain than on fact.

M. R. Hilleman and his colleagues ${ }^{2} 3$ at West Point, Pennsylvania, have shown that mumps vaccine prepared in duck embryo cell cultures was immunogenic, generally free from reactions, and conferred protection when an outbreak of mumps occurred shortly after administration of the vaccine. They have now studied combinations of measlesrubella-mumps ${ }^{4}$ and rubella-mumps vaccines. ${ }^{5}$ Studies on several hundred children who initially lacked antibody to all three viruses showed seroconversion rates of between 94 and 99\% against all antigenic components. The antibody levels were much the same as when the vaccines were administered separately. These studies indicate that simultaneous administration of combined vaccines is not accomoanied by interference of one vaccine with the other, and there was no increase in clinical reactions owing to the vaccines being given together. If a decision is taken to protect children, either individually or collectively, against these diseases, it is useful to know that the vaccines can be combined in a single dose, but it would seem that we need to know more about the long-term protective efficacy of a mumps vaccine before it is adopted as a routine measure for children. In the meantime it would appear to be a useful vaccine for adults who have escaped childhood infection.

' Mever, H. M., Hopps, H. E., Bernheim, B. C., and Douglas, R. D., in First International Conference on Vaccines against Viral and Rickettsial Diseases of Man, Washington, November, 1966, p. 336. Washington, Pan American Health Organization, 1967.

Weibel, R. E., Buynak, E. B., Stokes, J., Whitman, J. E., and Hilleman, M. R., in First International Conference on Vaccines against Viral and Rickettsial Diseases of Man, Washington, November, 1966, p. 430. Wickettsial Diseases of Man, Washington, November,

- Hilleman, M. R., Buynak, E. B., Weibel, R. E., and Stokes, J., New England fournal of Medicine, 1968, 278, 227.

- Buynak, E. B., Weibel, R. E., Whitman, J. E., Stokes, J., and Hilleman, M. R., Fournal of the American Medical Association, 1969, 207, 2259. -Weibel, R. E., et al., fournal of the American Medical Association, 1971, 216, 983.

\section{Malignant Disease and the Nephrotic Syndrome}

From time to time reports appear of patients suffering simultaneously from malignant disease and the nephrotic syndrome. In some the renal condition may be attributable to amyloidosis $^{1}$ or to renal vein thrombosis, ${ }^{2}$ and in others the association may be due to chance. However, J. C. Lee and his colleagues ${ }^{3}$ calculated that the association was much more frequent than would be expected on this basis, and it seems likely that there is some other connexion between the two conditions.

The possibilities would seem to be that both have a common cause, or that the nephrotic syndrome arises as a complication of the neoplasm. The latter hypothesis seems particularly likely in those rare cases in which treatment directed towards the neoplasm results in a remission of the nephrotic syndrome. In 1969 E. G. Cantrell ${ }^{4}$ reported on a patient whose nephrotic syndrome remitted after subtotal gastrectomy for carcinoma of the stomach, and recently $\mathrm{J}$. Plager and L. Stutzman 5 have reviewed reports of Hodgkin's disease associated with the nephrotic syndrome. They discovered ten cases and reported four more of their own. These four cases showed a remarkable temporal correlation between multiple relapses and remissions of the two conditions. In three cases investigations performed in life or at postmortem excluded the possibilities of amyloidosis or renal vein thrombosis, and in any case a relapsing nephrotic syndrome would be a very unusual manifestation of these disorders. Renal tissue was obtained by biopsy in two cases and at necropsy in two. The appearance of the material was reported to be within normal limits, but unfortunately it was not possible to examine it either by immunofluorescent techniques or by electron microscopy. Indeed, the extent of the examination by light microscopy is far from clear. However, many features of the patients' illnesses suggested that the renal lesions were minimal. Plager and Stutzman point out that much of the treatment they prescribed, particularly the steroid and cytostatic drugs, might be expected to benefit the renal disorder directly. But on several occasions remissions of both disorders followed short courses of radiotherapy applied to peripheral lymph nodes, and the authors conclude that the nephrotic syndrome occurring in association with Hodgkin's disease is likely to be due to renal damage induced either directly by a substance produced by the tumour or indirectly via an immunological reaction to the tumour. This could lead either to the formation of circulating antigen-antibody complexes, which might lodge in the kidney, or to renal damage due to cross-reaction of relatively non-specific antitumour antibodies with the glomerular basement membrane.

Hodgkin's disease is notorious for the multiplicity of its systemic manifestations, and it does not seem unreasonable to postulate the production by the tumour of a substance which could alter the permeability of the glomerular basement membrane without producing gross histological changes. On the other hand, glomerulonephritis produced experimentally by circulating antigen-antibody complexes or by antibody to glomerular basement membrane is associated with changes visible on ordinary light microscopy. 6 Thus participation of either of these mechanisms in the three cases having normal renal morphology seems to be rather unlikely. However, perhaps different mechanisms operate in different patients. Two of the patients from reports cited by Plager and Stutzman were said to have membranous nephropathy, and this appears to be the commonest renal lesion when the nephrotic syndrome is associated with carcinomata. ${ }^{3}$

Recently L. W. Loughridge and M. G. Lewis, from the Westminster Hospital, ${ }^{7}$ reported three more cases with malignant disease and the nephrotic syndrome. One of these, a man with carcinoma of the bronchus, had a membranous nephropathy, which was studied by immunofluorescent techniques. Deposits of IgG and IgM were found on the glomerular basement membrane. They had a "lumpy" appearance characteristic of that seen after the deposition of antigen-antibody complexes and unlike the "linear" appearance characteristic of nephritis produced by anti-kidney serum. Subsequently ${ }^{8}$ further (necropsy) material from the same patient was examined, and identical antigens were detected in an extract of the tumour and an eluate from the glomeruli.

Plager and Stutzman conclude their article by comparing their patients to the New Zealand black mice. Animals of this species develop a complex illness with glomerulonephritis and have a high incidence of lymphomata. A murine 
leukaemia-like virus has been thought to cause this illness, and viral antigenic components have been identified in the tumours and in the glomerular lesions. ${ }^{9}$ This comparison provides food for thought even though no viral cause for Hodgkin's disease has been identified and the renal disease in the mice is associated with pathological changes similar to membranous glomerulonephritis. ${ }^{10}$

1 Kimball, K. G., Annals of Internal Medicine, 1961, 55, 958.

Harrison, C. V., Milne, M. D., and Steiner, R. E., Quarterly fournal of Medicine, 1956, 25, 285.

- Lee, J. C., Yamauchi, H., and Hopper, J., Annals of Internal Medicine, $1966,64,41$.

( Cantrell, E. G., British Medical fournal, 1969, $2,739$.

Plager, J., and Stutzman, L., American Fournal of Medicine, 1971, 50, 56. Dixon, F. J., in Proceedings of the Third International Congress of Nephrology, vol. 2, ed. R. H. Heptinstall. Basel and New York, Karger, 1966. ' Loughridge, L. W., and Lewis, M. G., Lancet, 1971, 1, 256.

- Lewis, M. G., Loughridge, L. and Phillips, T. M., Lancet, 1971, 2, 134.

Mellors, R. C., Aoki, T., and Huebner, R. J., Fournal of Experimental Medicine, 1969, 129, 1045.

${ }^{10}$ Mellors, R. C., Fournal of Experimental Medicine, 1965, 122, 25.

\section{Misadventure}

Though it is running four years late, the Registrar General's Commentary on the statistics of England and Wales always provides some useful information not otherwise obtainable. Among other things it provides a useful check on the drugs particularly apt to cause serious adverse reactions. The deaths from this cause in 1967 numbered 146, an approximately average figure for the years 1962-7, though the variation was from 68 in 1964 to 179 in 1965. Deaths from anaesthetics showed a sharp rise from 1 in each of the years 1962 and 1963 to 17 in 1967 . The extent to which this increase is real and not due to more explicit reporting is hard to assess, but the Registrar General does draw attention to "a comparatively new feature" in deaths associated with attempted endotracheal anaesthesia. The tube has been known to enter the stomach and rupture it or to become obstructed, with resulting asphyxia or cardiac arrest.

Of drugs other than anaesthetics that caused death from adverse reactions the outstanding groups were corticosteroids (24 deaths) and phenylbutazone (14 deaths). In contrast to some high totals in previous years chloramphenicol caused only two deaths. For the first time contraceptive pills enter the list as a cause of death, two being attributed to them. The well-known hazards of the anticoagulants are reflected in the $\mathbf{1 5}$ deaths they caused. Six deaths from renal disease followed the administration of phenacetin, mainly for rheumatoid arthritis. Among surgical procedures as causes of death the most notable was blood transfusion, to which 14 deaths were attributed.

Among the oddities traditionally included in this volume is an interesting analysis of deaths from lightning. The majority of those killed are males, and the season at which most deaths occur is the summer. The average number of people killed per year has declined this century, from 12.4 per year in the decade 1901-10 to 9.6 in 1951-60 and 3.6 in 1961-7. The numbers of deaths may possibly have decreased, as the report suggests, because people congregate out of doors for work and play less than they used to do. Certainly the numbers of farm workers have diminished, but as the whole explanation this hardly carries conviction. It would be interesting to know whether lightning strikes as often as it did or whether some climatic change has turned Jove's attention elsewhere.

1 Registrar General's Statistical Review of England and Wales for the year 1967, Part III, Commentary. London, H.M.S.O., 1971 (£3.05 net).

\section{Health Care in the U.S.A.}

The B.M.A. came under friendly scrutiny last week when Senator Edward Kennedy and other members of the Senate Health Subcommittee investigating the N.H.S. had a private meeting with the Chairman of Council and a small group of doctors from the main branches of the profession. The Senator was particularly concerned with the provision of primary medical care, the problems of junior hospital staff, and the relationship between profession and government, and his team will be reporting its findings to the Congress later this year.

There are five different proposals for reform of the provision of medical care in the U.S.A., all at present in committee in Washington. One of these, the Kennedy plan, is for a comprehensive service financed partly by federal taxes and partly by payroll and income taxes-costing $\$ 41,000$ million a year; two other comprehensive schemes would be rather more expensive. The American Medical Association's Medicredit plan would provide only basic hospital and doctor care, while the fifth plan, the PettengillAetna proposal, would be limited to the uninsurable classes. The only certainty at the moment seems to be that some socialization of medicine will be introduced in the near future, but which plan will be approved is far from clear.

Earlier this year the Anglo-American Conference on Medical Care at the Royal Society of Medicine was told ${ }^{1}$ that "many Americans regarded the British N.H.S. as the worst in the world with the possible exception of the Russian one." Senator Kennedy's team has paid only a flying visitbut they should have seen enough to realize that a national health service in the British pattern is far better than its transatlantic critics would like to believe.

1 Lancet, 1971, 1, 789. 